\title{
"Trans-Localizando", una actividad lúdica para estudiar el problema de localización-ruteo
}

"Trans-Localizando", a hands on activity to study the location-routing problem

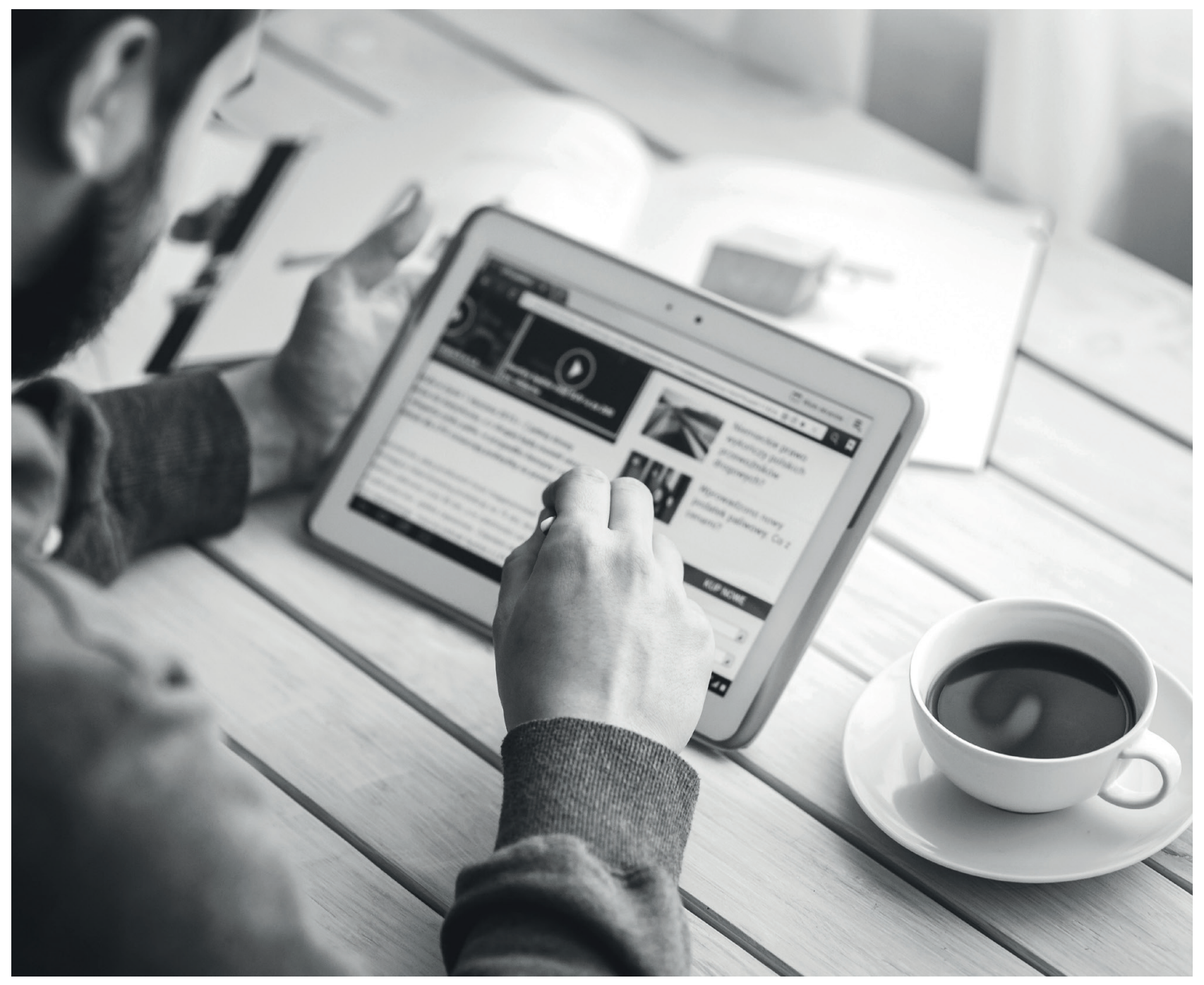

Fuente: Pexels.com Licencia Creative Commons 


\title{
"Trans-Localizando", una actividad lúdica para estudiar el problema de localización- ruteo $^{1}$
}

\author{
"Trans-Localizando", a hands on activity to study the location- \\ routing problem
}

\author{
Carolina Saavedra-Moreno ${ }^{2}$, Laura Daniela Marín Mendoza ${ }^{3}$
}

Artículo recibido en julio 17 de 2020; artículo aceptado en agosto 31 de 2020

\begin{abstract}
Este artículo puede compartirse bajo la Licencia Creative Commons Atribución-NoComercial-Compartirlgual 4.0 Internacional y se referencia usando el siguiente formato: Saavedra-Moreno, C., Marín, L. D. (2021). “Trans-Localizando” una actividad lúdica para estudiar el problema de localización-ruteo. I+D Revista de Investigaciones, 16(1), 126-135. http://dx.doi.org/10.33304/revinv.v16n1-2021011.
\end{abstract}

\begin{abstract}
Resumen
En este artículo se presenta una propuesta de actividad lúdica diseñada para el estudio del problema de localizaciónruteo, en el curso de Diseño de Cadenas de Suministro, del programa de Ingeniería Industrial de una universidad colombiana. Para la selección y diseño de la actividad, se llevaron a cabo las siguientes fases: priorización de las temáticas por medio del análisis de las variables grado de dificultad y grado de motivación; configuración de la lúdica y de su validación preliminar, para evaluar el grado de cumplimiento y pertinencia. La temática fue seleccionada por ser uno de los temas prioritarios de intervención, puesto que presentó el promedio más alto respecto al grado de dificultad y al grado de interés. Se obtiene una lúdica que simula una red de suministro de dos niveles y vincula tres decisiones: 1) determinar cuáles, de tres posibles mayoristas, deben ser abiertos para atender a cuatro minoristas; 2) asignar cada minorista a los mayoristas abiertos, y 3) definir las rutas de entrega de mercancía, minimizando costos en la red de suministro (inversión y transporte). Su estructura incluye información general relacionada con la asignatura, el contexto, los roles, el objetivo, los parámetros, las bonificaciones y las instrucciones detalladas que se deben llevar a cabo, la duración y los recursos requeridos para la ejecución de la actividad. En la etapa de validación todos los participantes manifestaron que la lúdica cumple el objetivo propuesto, es pertinente e interesante.
\end{abstract}

Palabras clave: Lúdica, modelo matemático, problema localización, ruteo.

\footnotetext{
'Artículo de investigación, de enfoque mixto, resultado de un trabajo de grado de pregrado culminado, titulado "Propuesta de actividades lúdicas para el apoyo de los procesos de enseñanza-aprendizaje de la asignatura Diseño de Cadenas de Suministro del programa de Ingeniería Industrial", perteneciente al área de ciencias sociales e ingenierías, subárea de operaciones y procesos, desarrollado en el grupo de investigación GINNOVA programa de Ingeniería Industrial, Universidad de Ibagué (Colombia). Dirección: carrera 22 calle 67, Ibagué - 730002. PBX: +57 (8) 2760010 . Fecha de inicio: julio de 2019, fecha de finalización: febrero de 2020.

2 Magíster en Gestión Industrial, Universidad de Ibagué. Vinculada al grupo de investigación GINNOVA, programa de Ingeniería Industrial, Universidad de Ibagué (Colombia). Dirección: carrera 22 calle 67, Ibagué - 730002. PBX: +57 (8) 2760010 . ORCID ID: https://orcid.org/0000-00026569-7932. Correo electrónico institucional: carolina.saavedra@unibague.edu.co.

${ }^{3}$ Estudiante de Ingeniería Industrial, Universidad de Ibagué. Vinculada al programa de Ingeniería Industrial. Universidad de Ibagué. Dirección: carrera 22 calle 67, Ibagué - 730002, Colombia. PBX: +57 (8) 276 0010. ORCID ID: https://orcid.org/0000-0002-9299-2785. Correo electrónico institucional: 2320152020@estudiantesunibague.edu.co.
} 
Carolina Saavedra-Moreno, Laura Daniela Marín Mendoza

"Trans-Localizando" una actividad lúdica para estudiar el problema de localización-ruteo

\begin{abstract}
This paper presents a proposal of a playful activity designed for studying the location-routing problem, in the course of Diseño de Cadenas de Suministro, in an Industrial Engineering program at a Colombian university. For the selection and design of the playful activity, the following phases were developed: prioritization of the themes through the analysis of the degree of difficulty and degree of motivation variables, configuration of the playful activity and the preliminary validation to assess the degrees of compliance and relevance. The theme was selected as one of the priority topics for intervention since it presented the highest mean value for the degree of difficulty and the degree of interest. The playful activity simulates a supply chain with two levels and includes the following decisions: 1) to determine which (out of three options) wholesalers should open to serve four retailers; 2 ) to allocate retailers to the open wholesalers, and 3) to deliver routes that start and finish in each wholesaler, so that the costs of the supply network (investment and transport) are minimized. The structure of the activity includes general information (related to the subject), context, roles, objective, parameters, bonuses, detailed instructions, duration, and resources required. In the validation phase, all the participants considered that the game met the goal, it was relevant and interesting.
\end{abstract}

Keywords: Playful activity, mathematical model, location problem, routing.

\section{Introducción}

Los modelos educativos tradicionales se rigen a partir de procesos analíticos, donde sobresale el pensamiento lógico, estructurado, lineal y progresivo. Este es indispensable para el aprendizaje. Sin embargo, estos patrones de enseñanza limitan la visión global e integral que se puede tener de los problemas en un contexto real $y$, por ende, las habilidades necesarias para solucionarlo (Villa Sánchez y Poblete Ruiz, 2011). A razón de lo anterior, se han venido implementando, en los diferentes niveles de formación, nuevas metodologías de enseñanza y aprendizaje, como el aprendizaje basado en proyectos $(A B P)$, el aprendizaje basado en juegos (ABJ), entre otros (Méndez Coca, 2011). Estas nuevas tendencias de enseñanza y aprendizaje permiten que los estudiantes adopten un rol activo (Acosta-Medina et al., 2020), desde el cual logran un conocimiento significativo; y de forma paralela, aumentan el interés y la actitud por las temáticas de estudio. Particularmente, el $A B J$ le brinda al estudiante un ambiente propicio para aprender a aprender y le da la posibilidad de equivocarse, asumir roles, llegar a múltiples respuestas, asumir retos y recibir una realimentación conforme a los resultados obtenidos (Shute y Ke, 2012).

Una de las asignaturas más importantes en la línea de profundización del programa de Ingeniería Industrial de la universidad colombiana objeto de estudio es el curso Diseño de Cadenas de Suministro. Este curso tiene como objetivo principal el estudio de las decisiones estructurales de planificación de la cadena de suministro. Su importancia radica en la relevancia de sus temáticas para la formación disciplinar del ingeniero industrial. Teniendo en cuenta que en la asignatura se evidenció un alto componente de clases magistrales y bajo interés de los estudiantes hacia algunos tópicos del curso, se decidió diseñar actividades lúdicas en el marco del $A B J$ para los tópicos de mayor prioridad. El objetivo de las propuestas fue disminuir la percepción de dificultad de los estudiantes, aumentar el grado de interés y mejorar sus resultados académicos.

Unas de las temáticas priorizadas fueron los modelos integrados de optimización en el diseño de cadenas de abastecimiento, específicamente el problema de localización-ruteo. Escobar et al. (2015) y Laporte (1987) indican que aunque la localización es una decisión estratégica, tomada por un largo periodo de tiempo, y el ruteo es una decisión operacional que funciona dinámicamente y puede ser modificada varias veces en un periodo corto, ambas decisiones están relacionadas, debido a que la localización de un depósito es a menudo influenciada por los costos de transporte, y viceversa. De allí que sea muy importante el estudio conjunto de estas decisiones.

En la literatura hay diversidad de actividades lúdicas relacionadas con logística y gestión de las cadenas de suministro. Por un lado, se encuentran lúdicas que recrean cadenas de suministro. Una de las más populares es el Juego de la Cerveza, una actividad creada por un grupo de profesores del Sloan School of Management del MIT, que tiene por objetivo mostrar principios claves de la gestión de la cadena de suministro (Sterman, 1989). Lau (2015) plantea una modificación al Juego de la Cerveza, incluyendo escenarios, tales como el rediseño de la cadena, la reducción del tiempo de entrega, la visibilidad de la información y la posibilidad de pronósticos colaborativos. Sarmiento y Rodríguez (2019) proponen una lúdica denominada "The Supply Chain Game", cuyo objetivo es presentar los conceptos generales de las cadenas de suministro y las implicaciones económicas de las decisiones asociadas.

Particularmente en los temas de localización y ruteo, en la literatura se identifican lúdicas que analizan cada una de las decisiones de manera independiente. Por ejemplo, Carvajal etal. (2019) proponen una lúdica para el problema de ruteo de vehículos denominada "¡Pizza caliente ya!". El objetivo de esta actividad es proponer las rutas de 
entrega de las pizzas usando el método de ahorros de Clark y Wright, y teniendo en cuenta restricciones de tiempo y distancia. Con respecto a la localización, William et al. (2018), en su juego de mesa relacionado con la gestión de cadenas de suministro humanitarias y urbanas, vinculan la decisión de determinar la ubicación óptima de los almacenes, teniendo en cuenta diferentes tipos de criterios de índoles cuantitativas y cualitativas.

En este artículo se presenta una actividad lúdica que integra las decisiones de localización y ruteo. La lúdica recrea una red de distribución de café liofilizado, con tres posibles centros de acopio que se pueden abrir para atender a cuatro minoristas. Poner en marcha cada uno de los centros de acopio vincula unos requerimientos de inversión. Además, se incluyen los costos de transporte entre eslabones. El objetivo de la actividad es que los estudiantes identifiquen claramente los componentes del modelo de optimización que permita definir cuál o cuáles centros de acopio deben abrirse, la asignación de los minoristas a cada centro de acopio abierto y las rutas de entrega de producto, de tal manera que se minimicen los costos totales en la red de suministro (inversión y transporte).

\section{Marco referencial}

El problema de localización-ruteo (LRP, por sus siglas en inglés) combina dos decisiones estructurales del diseño de cadenas de abastecimiento: la ubicación de instalaciones y el transporte (abastecimiento desde rutas) (Saracche y Morales, 2016). Dado un conjunto de instalaciones potenciales con costos de apertura, una flota de vehículos homogéneos y un conjunto de clientes con demandas conocidas, el LRP clásico establece las instalaciones que se abrirán, la asignación de clientes a estas y la definición de rutas para cada instalación y sus clientes, de tal manera que se minimice el costo total (este costo incluye el costo de abrir las instalaciones, los costos de transporte asociados a cada ruta y los costos de los vehículos utilizados) (Karaoglan y Altiparmak, 2015; Linfati et al., 2014).

Prins et al. (2007) formularon el problema LRP así: sea $G$ $=(V, E)$ un grafo no dirigido, donde $V=1, \ldots, m+n$ es un conjunto de nodos que incluye un subconjunto I de $m$ posibles localización para instalarse y un subconjunto $J$ de $n$ clientes. $E$ corresponde al conjunto de arcos entre eslabones. Las instalaciones tienen una capacidad $W$ y un costo de apertura $O$. Cada cliente $j$ tiene una demanda $d$ conocida, y el costo de viajar entre dos nodos $i$ y $j$ viene dada por $C_{i j}$. A su vez, un conjunto $k$ de vehículos homogéneos de capacidad fija $Q$ cuando realiza una ruta genera un costo fijo $F$ y unos costos variables de acuerdo con la ruta. El problema se modela como un modelo de programación lineal entero binario con variables de decisión binarias asociadas a la apertura de la instalación, la asignación de un cliente a una instalación y la utilización de un arco de $i$ a j en la ruta asignada a un vehículo $k$.
La función objetivo comprende los costos de las instalaciones abiertas, los costos de los arcos visitados por las rutas y los costos fijos de los vehículos utilizados. Las restricciones incluidas son:

- Cada cliente es atendido por una de las instalaciones, pertenece a una sola ruta y en esta tiene un único predecesor.

- Las cantidades por entregar en cada ruta asignada a una instalación no superan las capacidades de los vehículos y de la instalación, respectivamente.

- Se debe garantizar la continuidad de cada ruta, así como que cada una de ellas inicie y termine en la misma instalación y sea atendida por un solo vehículo.

- Un cliente será asignado a una instalación siempre y cuando exista una ruta que lo una con esta.

\section{Metodología}

Este estudio es de tipo descriptivo y propositivo, con enfoque cualitativo. Las fases que se llevaron a cabo para el desarrollo fueron la priorización de las temáticas, el diseño de la lúdica y la validación preliminar.

En primera instancia se realizó un proceso de priorización de temáticas, estudiando las variables grado de dificultad y grado de interés, por medio de una encuesta estructurada en dos partes. En la primera parte los participantes calificaron, según su percepción, en una escala de 1 a 5 , el grado de dificultad de cada una de las temáticas del curso (siendo $5=$ mayor grado de dificultad, y $1=$ menor grado) y el grado de interés o motivación (siendo $5=$ alto grado de interés, y $1=$ bajo grado). En la segunda parte, a partir de preguntas abiertas, se recolectaron las razones de las calificaciones dadas y las estrategias que se podrían implementar para disminuir la dificultad e incrementar la motivación. La encuesta se aplicó a una muestra de 20 participantes, conformada por 3 docentes del área y 17 estudiantes que habían cursado la asignatura (con estado aprobado y reprobado). La encuesta se compartió vía correo institucional por medio del recurso de Google Forms.

En esta primera fase, como segunda medida, se analizaron las relaciones entre las variables grado de dificultad y grado de interés, a través de una matriz de priorización que se construyó teniendo en cuenta las asociaciones entre las variables (ver Figura 1). La matriz se compone de cuatro cuadrantes, cuyos nombres representan el tipo de estrategias que se deben utilizar para mejorar el desempeño de la temática en las variables evaluadas. Los cuadrantes son:

- Motivación: Temas que en promedio tienen percepción de baja dificultad y bajo interés.

- Éxito: Es el cuadrante ideal. Tópicos que se perciben con un bajo grado de percepción de dificultad y alto grado de interés.

- Crítico: Temas que se perciben con alta percepción de 
Carolina Saavedra-Moreno, Laura Daniela Marín Mendoza

"Trans-Localizando" una actividad lúdica para estudiar el problema de localización-ruteo

dificultad y al mismo tiempo un alto nivel de interés.

- Prioridad: Temáticas que, además de tener un bajo nivel de interés, poseen un alto grado de dificultad.

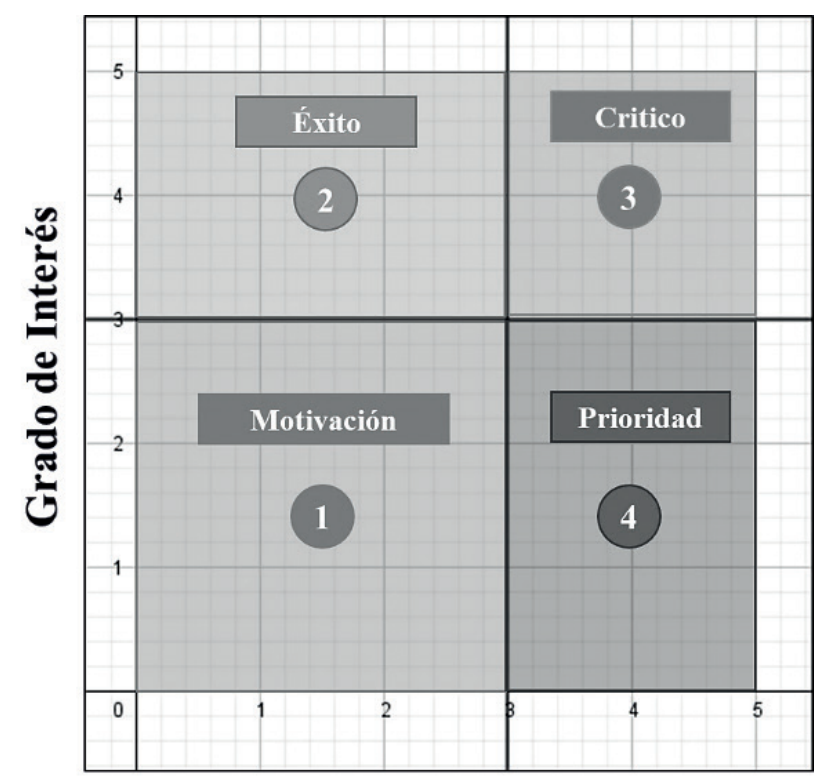

Grado de Dificultad

Figura 1. Matriz de priorización. Fuente: Autores.

En la fase de diseño de la lúdica se determinó la estructura de la actividad lúdica para el tema, teniendo en cuenta los principios de diseño de juegos recopilados en Contreras y Eguia (2017). La estructura vincula los siguientes elementos:

- Información general: asignatura, tema y objetivos de aprendizaje.

- Narrativa: se presenta el contexto real en el que se lleva a cabo la actividad, los roles de cada participante, el objetivo de la lúdica, los parámetros de entrada, las bonificaciones e instrucciones detalladas que se deben llevar a cabo.

- Recursos y tiempo: materiales, formatos y duración estimada.

Finalmente, se realizó una validación preliminar con un grupo de 5 participantes, cuatro estudiantes de octavo semestre del programa de Ingeniería Industrial y una docente del área de Procesos y Operaciones, en el Laboratorio de Ingeniería Industrial de la universidad donde se desarrolló la lúdica. En el encuentro, los asistentes llevaron a cabo la actividad siguiendo las instrucciones entregadas (ver Figura 2). Durante el desarrollo se documentaron las preguntas que se generaron al leer los documentos, las dificultades para comprender las instrucciones y, al final, se consultó a los participantes su percepción respecto al grado de motivación y el grado de cumplimiento del objetivo de la lúdica. Tomando como base la experiencia del encuentro y las opiniones de los invitados, se realizaron los ajustes pertinentes en la propuesta final de la lúdica.
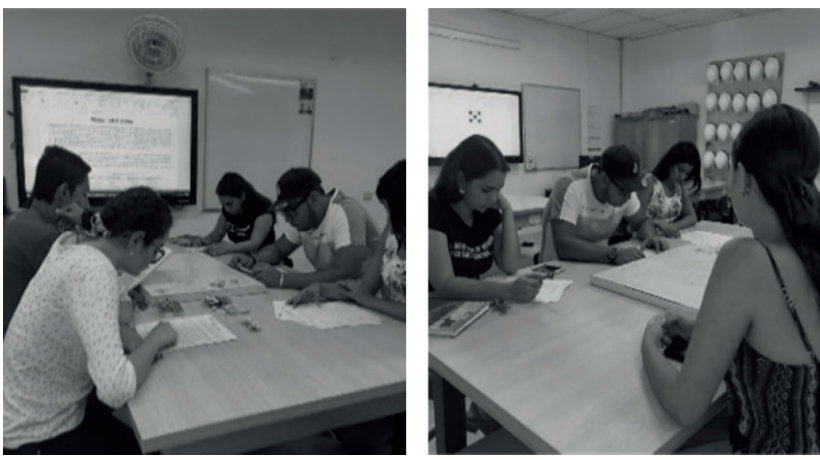

Figura 2. Registro fotográfico validación preliminar. Fuente: Autores.

\section{Resultados}

\section{Priorización de las temáticas}

En la Figura 3 se observa la clasificación de cada una de las temáticas en la matriz de priorización. Se evidencia que ninguna de las temáticas se localizó en los cuadrantes Motivación y Prioridad. Es de resaltar que los promedios de la variable Grado de interés estuvieron por encima de la puntuación tres. De otra parte, el $79 \%$ de los temas del curso se ubican en el cuadrante Éxito, y el $21 \%$, en el cuadrante Crítico. En este último se clasificaron los tópicos de modelos integrados de optimización para el diseño de cadenas de abastecimiento (MIO), distribución de un almacén (DA), modelos de inventarios multiproducto (MMP) y el problema de ruteo de vehículos (PRV).

Tal y como se evidencia en la Figura 3, el tópico modelos integrados de optimización para el diseño de cadenas de abastecimiento, representado por las siglas $\mathrm{MIO}$, fue el que presentó el promedio más alto respecto al grado de dificultad y el valor medio más alto de interés. Teniendo en cuenta que dentro de los modelos integrados existen varios tipos de problemas, y que el problema de ruteo de vehículos era otro de los temas del cuadrante crítico, se seleccionó particularmente el problema de localizaciónruteo para el diseño de la actividad lúdica.

\section{Diseño de la lúdica "Trans-Localizando"}

\subsection{Información general}

- Asignatura: Diseño de Cadenas de Suministro

- Tema: Modelos integrados de optimización para el diseño de las cadenas de abastecimiento: problema localización-ruteo

- Objetivos de aprendizaje:

- Identificar los elementos del modelo matemático del problema de localización-ruteo.

- Reconocer la modelación matemática como una herramienta útil, al momento de estudiar las decisiones de diseño de cadenas de suministro. 
Carolina Saavedra-Moreno, Laura Daniela Marín Mendoza "Trans-Localizando" una actividad lúdica para estudiar el problema de localización-ruteo

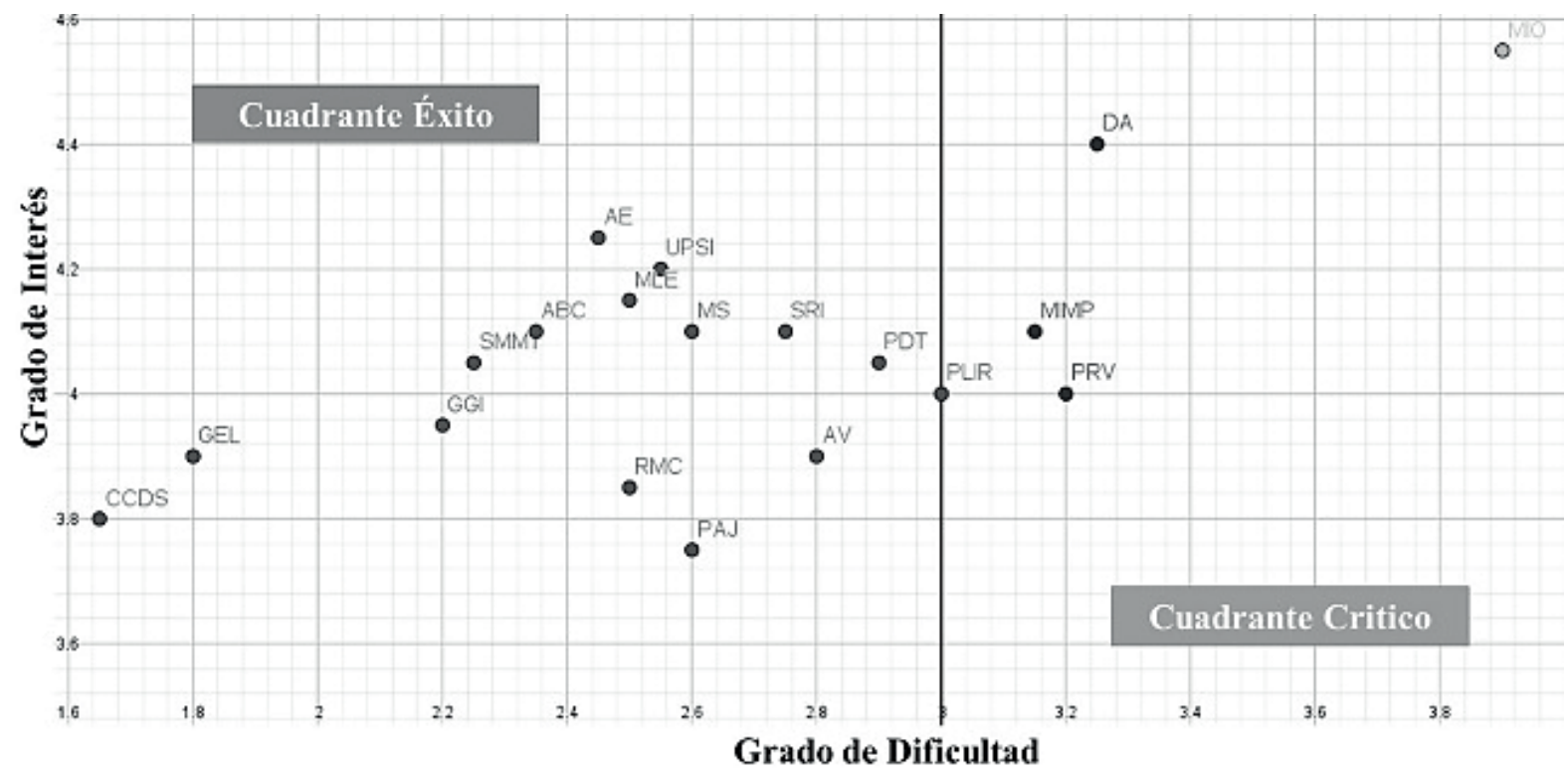

Figura 3. Priorización de las temáticas. Fuente: Autores.

\subsection{Narrativa}

- Contexto: se presenta la información para el diseño de una cadena de suministro de dos eslabones (centros de acopio-minoristas), del sector agroindustrial cafetero del departamento del Tolima. Hay tres posibles centros de acopio que se pueden abrir y están ubicados en los municipios de Líbano, Planadas y Cajamarca. Los centros de acopio abiertos deben atender a cuatro minoristas ubicados en los municipios de Alvarado, Ataco, San Antonio e Icononzo (ver Figura 4).

- Roles: cada equipo está conformado por cuatro estudiantes. Se requieren dos planeadores y dos agentes ejecutores. Los planeadores, por un lado, analizarán los datos de entrada y propondrán una alternativa de solución; por otro lado, los agentes ejecutores implementarán la propuesta realizada por los planeadores. Durante el juego se espera que los roles cambien por lo menos una vez; es decir, que se evalúen dos escenarios. Todos los participantes estiman los costos de cada estrategia y finalmente formulan el modelo matemático que permita encontrar la solución óptima. Teniendo en cuenta que se espera realizar comparación entre los resultados, se requiere tener mínimo dos equipos. El máximo número de equipos depende de la disponibilidad de los materiales requeridos y el número de docentes a cargo. Para facilitar el acompañamiento y asesoría del profesor, se recomienda máximo 6 equipos por profesor.

- Objetivo de la lúdica: la lúdica vincula dos objetivos. El primero, de índole práctico, es determinar las tres decisiones estudiadas en el problema de localización-ruteo: definir cuál o cuáles centros de acopio deben abrirse, qué oferente abastecerá a cada cliente minorista y las rutas de atención desde cada centro de acopio hacia los clientes, de tal manera que se obtenga el menor costo posible. Por otro lado, a partir de la experiencia se espera que los estudiantes conceptualicen el modelo matemático que permite encontrar la respuesta óptima al problema definido.

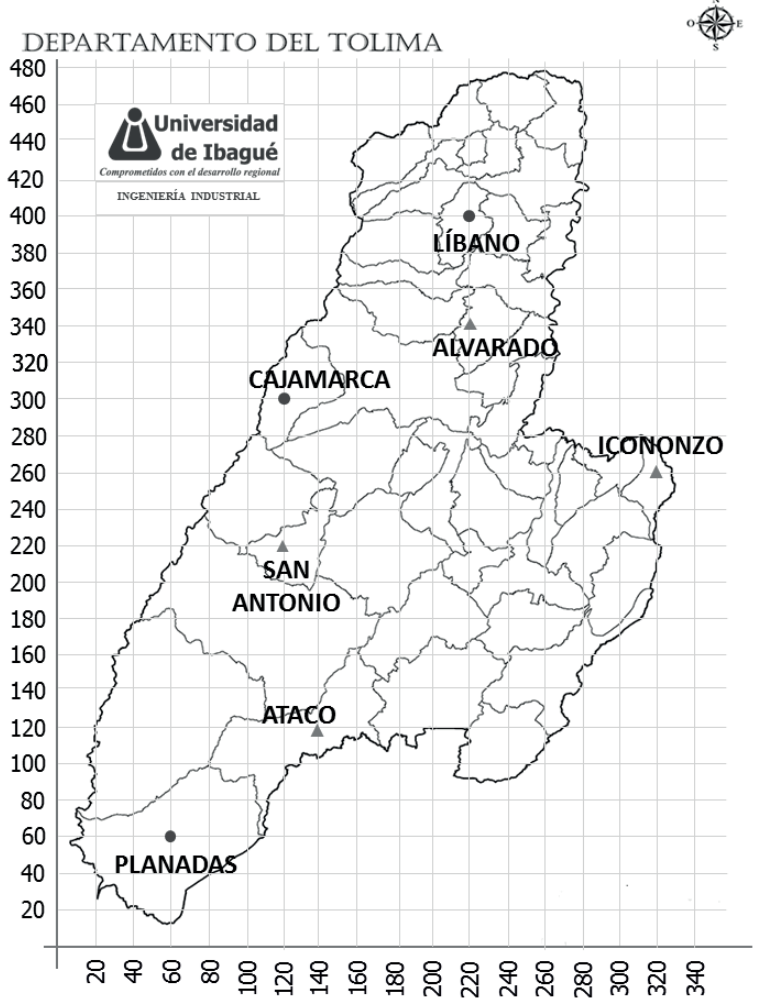

Figura 4. Tablero mapa político del Tolima, Juego Trans-Localizando. Fuente: Adaptado de https://www.bibliocad.com/es/biblioteca/mapadel-tolima-_85686/. 
Carolina Saavedra-Moreno, Laura Daniela Marín Mendoza

"Trans-Localizando" una actividad lúdica para estudiar el problema de localización-ruteo

- Parámetros de entrada

- Capacidad de centros de acopio

- Costos de apertura de los centros de acopio

- Distancia rectilínea entre eslabones teniendo en cuenta coordenadas en el mapa

- Costos de transporte unitario entre todos los eslabones de la red

- Costos fijos de cada ruta

- Capacidad de los vehículos

- Número de vehículos disponibles (solo se incluye un tipo de vehículo)

- Demanda de los minoristas

- Bonificaciones: se tienen dos tipos de bonificaciones. Por un lado, una bonificación para quien proponga el escenario que genere el menor costo total en la red de suministro. Por otro lado, bonificaciones en dinero que se descuentan del costo total de la alternativa propuesta por cada equipo, dependiendo de si formulan adecuadamente las variables, la función objetivo y las restricciones del problema.

- Instrucciones generales:

a) Los estudiantes conformarán grupos de 4 participantes y asignarán los roles en el equipo.

b) El docente entregará la demanda estimada de cada minorista.

c) Los planeadores de cada uno de los equipos formularán una estrategia de solución en el Formato 1 (parte sombreada) que se presenta en el Figura 5.

d) Los agentes ejecutores indicarán en el mapa (ver Figura 4) el centro de distribución que se abrirá (utilizando los banderines correspondientes) y, con cordón y alfileres, trazarán las rutas de entrega de mercancía. Además, asignarán el vehículo que atenderá cada una de las rutas. Todas las rutas inician y terminan en cada centro de acopio abierto.

e) Todo el equipo estimará los costos de la estrategia en el Formato (parte sin sombra) que se presenta en la Figura 5.

f) Se repiten las instrucciones desde b hasta e.

g) Los estudiantes seleccionarán el escenario menos costoso para el concurso, que entrega la primera bonificación del juego al equipo que tenga la estrategia de menor costo.

h) Finalmente, los estudiantes formularán el modelo matemático usando el Formato 2, que se muestra en la Figura 6.

i) El docente presentará una propuesta de modelo matemático, y compararán las respuestas con los estudiantes.

j) Cada equipo restará de sus costos totales los valores de bonificación obtenidos por la adecuada formulación del modelo.

k) Cada grupo, por medio de un vocero, indicará el costo total obtenido.

I) El equipo con menor costo total presentará a la clase su propuesta, indicando todas las consideraciones que tuvieron en cuenta.

m) El docente realizará la realimentación final.

\subsection{Recursos y duración}

Para el desarrollo del juego se requieren los siguientes recursos:

- 1 tablero mapa político del Tolima (ver Figura 4)

- 3 banderines

- 1 caja de chinchones plastificados para sostener el cordón que representa las rutas.

- 1 metro de cordón

- Formatos para recolección de información que se presentan en las Figuras 5 y 6

La lúdica tiene una duración aproximada de 2 horas.

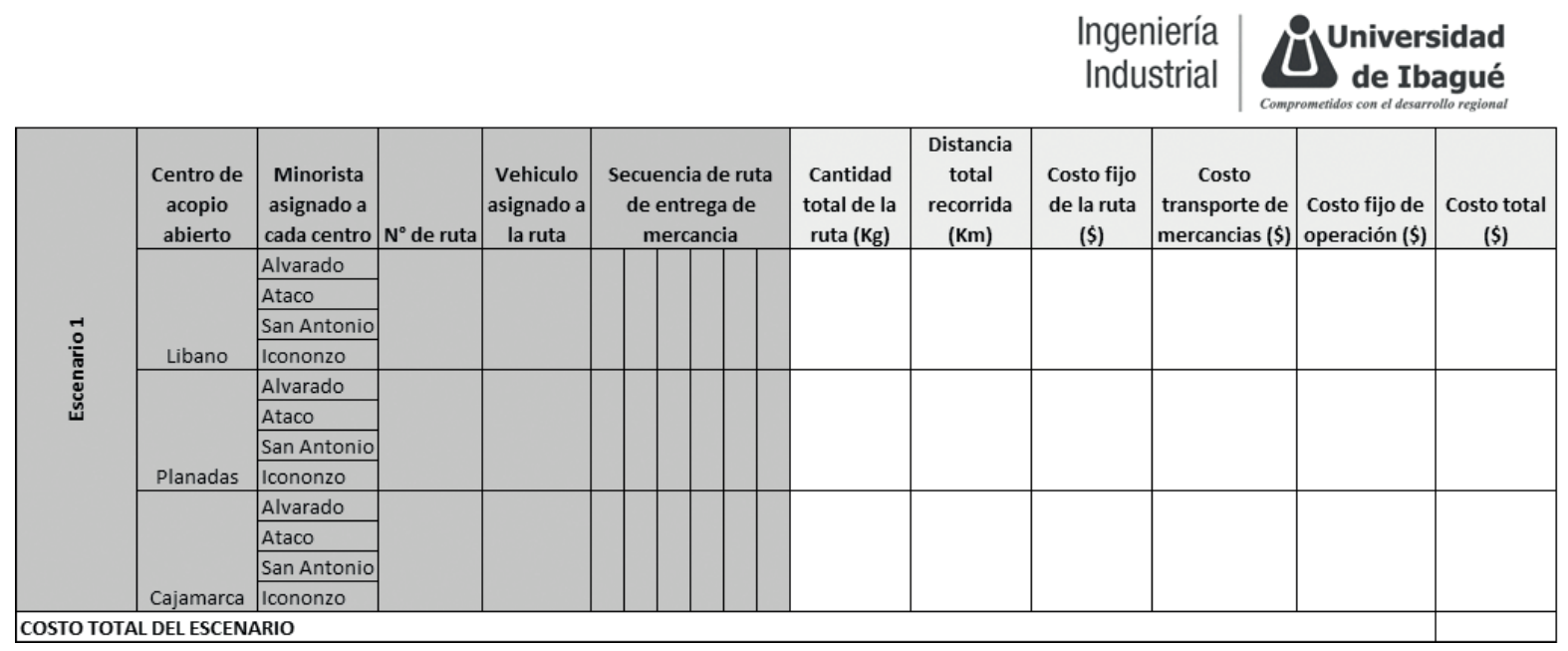

Figura 5. Formato construcción y evaluación de escenarios. Fuente: Autores. 


\section{Formato construcción del modelo matemático}

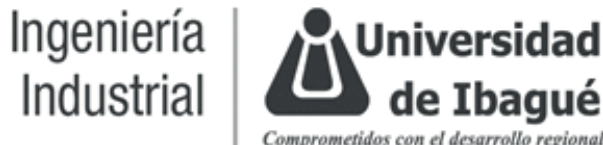

\begin{tabular}{|c|c|}
\hline $\begin{array}{l}\text { Revise los formatos de evaluación de escenarios, ¿Cuáles } \\
\text { fueron los valores que cambiaron en cada uno de ellos y les } \\
\text { permitieron encontrar nuevas soluciones? }\end{array}$ & $\begin{array}{l}\text { Especifique y clasifique las particularidades de la } \\
\text { situación propuesta que limitaron las soluciones } \\
\text { formuladas. }\end{array}$ \\
\hline Determine las variables del problema: & 1. Relacionados con los centros de acopio: \\
\hline $\begin{array}{l}\text { ¿Cual es el objetivo que se debia alcanzar al proponer los } \\
\text { escenarios? }\end{array}$ & 2. Relacionados con los minoristas: \\
\hline \multirow[t]{2}{*}{ Formule la función objetivo: } & 3. Relacionados con las rutas: \\
\hline & $\begin{array}{l}\text { Formule matemáticamente las restricciones planteadas } \\
\text { en la pregunta anterior: }\end{array}$ \\
\hline
\end{tabular}

Figura 6. Formato guía formulación modelo matemático. Fuente: Autores.

\section{Validación preliminar}

A partir de las observaciones socializadas por los participantes, se incluyeron las columnas relacionadas con las rutas en el formato de construcción y evaluación de los escenarios (ver Figura 5). El $100 \%$ de los participantes manifestó que la lúdica cumple el objetivo propuesto, es pertinente e interesante. Estos participantes resaltaron que para lograr el objetivo es importante que los estudiantes hubiesen cursado previamente las asignaturas de Investigación de Operaciones, para tener conocimientos básicos de modelación matemática. Además, consideraron necesario que, antes de aplicar la lúdica, en el curso de Diseño de Cadenas de Suministro ya se hubiesen abordado los modelos de optimización de las decisiones individuales de localización y ruteo. Uno de los invitados sugirió incluir las bonificaciones propuestas por acertar en los elementos del modelo matemático (función objetivo, variables de decisión y restricciones) como una bonificación monetaria, de tal forma que la puntuación obtenida se pueda restar de los costos inicialmente calculados.

La docente invitada recomendó que para utilizar la lúdica dentro del horario de clases es necesario que se evalúen tan solo dos escenarios de localización -asignaciónruteo, a razón de la limitación de tiempo. Finalmente, dos participantes mencionaron la importancia del rol del docente no solo como guía sino también como motivador.

\section{Discusión y conclusiones}

La propuesta metodológica presentada para categorizar las temáticas resulta útil para cualquier asignatura que necesite realizar una priorización de los tópicos que requieren el diseño de nuevas estrategias educativas. Lo anterior, desde el análisis de las variables Grado de dificultad y Grado de interés. Esta metodología permite al docente clasificar las temáticas en cuatro cuadrantes y diseñar estrategias para mejorar la percepción de los estudiantes en las variables medidas.

En la fase de priorización se encontró que en su mayoría (el $78 \%$ ) las temáticas abordadas en el curso se perciben como temáticas con bajo grado de dificultad, y los estudiantes presentan un alto interés hacia estas. Teniendo en cuenta que ninguna de las temáticas se ubicó en el cuadrante cuatro (de prioridad), la priorización se decantó por las temáticas ubicadas en el cuadrante crítico (alto grado de dificultad y alto grado de motivación).

"Trans-localizando" es una lúdica que permite al estudiante reconocer la modelación matemática como una herramienta útil, al momento de estudiar las decisiones de diseño de cadenas de suministro, particularmente en el problema localización-ruteo. La actividad le permite identificar los elementos del modelo matemático a partir de la evaluación de escenarios de solución para definir cuál o cuáles centros de distribución deben abrirse, qué oferente abastecerá a cada cliente minorista y la rutas que indicarán el orden de atención 
Carolina Saavedra-Moreno, Laura Daniela Marín Mendoza

"Trans-Localizando" una actividad lúdica para estudiar el problema de localización-ruteo

a clientes, con el objetivo de minimizar los costos totales de la red de distribución.

Una de las lúdicas más reconocidas en el tema objeto de análisis es el Juego de la Cerveza, una actividad en donde se recrea una cadena de suministro y se demuestran principios relevantes en la gestión de esta. Por otro lado, las lúdicas particulares de las temáticas de localización y ruteo se han centrado en estudiar cada tema de manera independiente, y se han enfocado en abordar metodologías de solución para cada uno de los problemas. "Trans-Localizando" es una lúdica que integra las dos decisiones, lo que facilita que el estudiante pueda comprender las relaciones entre estas y la importancia de estudiarlas de manera conjunta.

Al ejecutar la lúdica se encontraron barreras relacionadas con requerimientos de conocimientos previos y la necesidad de contar con formatos para registrar la información que se obtiene durante la ejecución del juego. Respecto a los conocimientos previos, se identificó que para participar en la actividad se deben tener conocimientos en modelación matemática básica, específicamente de los problemas de optimización propuestos para las decisiones de localización y ruteo, de manera independiente. Respecto a los formatos, luego de la validación se hicieron los ajustes recomendados, con el fin de que los estudiantes cuenten con las herramientas para registrar toda la información necesaria para costear las alternativas y documentar las partes del modelo matemático.

\section{Referencias}

Acosta-Medina, J. K., Torres-Barreto, M. L., ÁlvarezMelgarejo, M. y Paba-Medina, M. C. (2020). Gamificación en el ámbito educativo: Un análisis bibliométrico. I+D Revista de Investigaciones, 15(1), 30-39. https://doi.org/10.33304/revinv.v15n1-2020 003

Carvajal, N., Padilla, S. y Rocha, J. (2019). ¡Pizza caliente ya! Método de Clark and Wright para ruteo de vehículos. VII Encuentro de La Red IDDEAL. https:// peewah.co/events/septimo-encuentro-nacionalred-iddeal

Contreras Espinosa, R. S. y Eguia, J. L. (2017). Experiencias de gamificación en aulas. InCom-UAB Publicacions.

Escobar, J. W., Linfati, R. y Adarme Jaimes, W. (2015). Problema de localización y ruteo con restricciones de capacidad: Revisión de la literatura. Facultad de Ingeniería, 24(39), 85-98.

Karaoglan, I. y Altiparmak, F. (2015). A Memetic Algorithm for the Capacitated Location-Routing Problem with Mixed Backhauls. Computers \& Operations
Research, 55, 200-216. https://doi.org/10.1016/j.cor. 2014.06.009

Laporte, G. (1987). Location-Routing Problems. https:// www.osti.gov/etdeweb/biblio/6622976

Lau, A. K. (2015). Teaching Supply Chain Management Using a Modified Beer Game: An Action Learning Approach. International Journal of Logistics Research and Applications, 18(1), 62-81. https://doi.org/10.108 $0 / 13675567.2014 .945398$

Linfati, R., Escobar, J. W. y Gatica, G. (2014). Un algoritmo metaheurístico para el problema de localización y ruteo con flota heterogénea. Ingeniería y Ciencia, 10(19), 55-76. https://doi.org/10.17230/ing ciencia.10.19.3

Méndez Coca, D. (2011). Didáctica y aprendizaje de los conceptos básicos de la termodinámica [tesis de doctorado, Universidad Complutense de Madrid] Repositorio institucional: https://eprints.ucm. es/14722/

Prins, C., Prodhon, C., Ruiz, A., Soriano, P. y Wolfler Calvo, R. (2007). Solving the Capacitated Location-Routing Problem by a Cooperative Lagrangean RelaxationGranular Tabu Search Heuristic. Transportation Science, 41(4), 470-483. https://doi.org/10.1287/ trsc.1060.0187

Saracche, W. y Morales, M. (2016). Localización, transporte e inventarios. Tres decisiones estructurales en el diseño de cadenas de abastecimiento. Universidad Nacional de Colombia.

Sarmiento, J. y Rodríguez, V. (2019). The Supply Chain Game. VII Encuentro de La Red IDDEAL. https:// peewah.co/events/septimo-encuentro-nacionalred-iddeal

Shute, V. J. y Ke, F. (2012). Games, Learning, and Assessment. En D. Ifenthaler, D. Eseryel y X. Ge (eds.), Assessment in Game-Based Learning (pp. 4358). Springer. https://doi.org/10.1007/978-1-46143546-4 4

Sterman, J. D. (1989). Modeling Managerial Behavior: Misperceptions of Feedback in a Dynamic Decision Making Experiment. Management Science, 35(3), 321-339. https://doi.org/10.1287/mnsc.35.3.321

Villa Sánchez, A. y Poblete Ruiz, M. (2011). Evaluación de competencias genéricas: Principios, oportunidades y limitaciones. 63(1), 147-170.

William, L., Abdul Rahim, Z. Bin, De Souza, R., Nugroho, E. y Fredericco, R. (2018). Extendable Board Game to Facilitate Learning in Supply Chain 
Carolina Saavedra-Moreno, Laura Daniela Marín Mendoza

"Trans-Localizando" una actividad lúdica para estudiar el problema de localización-ruteo

Management. Advances in Science, Technology and

Engineering Systems Journal, 3(4), 99-111. https://

doi.org/10.25046/aj030411

I+D Revista de Investigaciones ISSN 2256-1676 / ISSN en línea 2539-519X

Volumen 16 Número 1 Enero-Junio de 2021 pp.126-135 